\title{
Pharmaceutical Care of the Patients Suffering from Depression
}

\author{
Zahida Binakaj and Svetlana Stojkov \\ Faculty of Pharmacy, University of Tuzla, Univerzitetska 8, 75000 Tuzla, Bosnia and Herzegovina
}

\begin{abstract}
Depression is the most present mental disorders with a very expensive treatment costs. Due to the high prevalence of depression in Bosnia and Herzegovina and high work load of psychiatrists, patients that suffer from depression are often marginalised, stigmatised and inadequately treated. Therefore, a pharmacist can influence an adequate selection of a therapy through an interdisciplinary approach and pharmaceutical care and alos provide patient monitoring, counselling about side effects and interactions of the prescribed drugs, which will in the end improve the quality of life for patients that suffer from depression. The aim of this work was to establish the influence of pharmaceutical care on the outcome of the quality of life and the pharmacological treatment of patients suffering from depression. Results showed that the involvement of pharmacist in a multidisciplinary approach to the treatment of depression lead to improvement of the effect of therapy and increased quality of life.
\end{abstract}

Key words: Depression, pharmaceutical care, quality of life.

\section{Introduction}

Depression is mental disorders characterised as a disorder of mood (unipolar disorder). It is a severe psychophysical state that is accompanied with various side effects. Depressive mood is characterised with the withdrawal behaviour, despondency, drop of vital signs, insomnia, loss of appetite, pessimism, difficulty concentrating and remembering details, hopelessness and helplessness [1]. Depression is a widespread disorder and is one of the earliest described illnesses in the history of medicine. According to the data of World Health Organisation more that $10 \%$ of world's population is suffering from depression. It is considered that the number of affected is much higher as many patients do not recognise that they are suffering and do not ask for help. In most cases depression occurs before 40 years of age and even more young people are becoming affected by this disorder.

There are large variations in the clinical picture of

Corresponding author: Zahida Binakaj, Faculty of Pharmacy University of Tuzla Univerzitetska 8. 75000 Tuzla, Bosnia and Herzegovina and Svetlana Stojkov, Pharmaceutical Chamber of Serbia. depression. There is range of theoretical explanations of its ethnology which includes genetic theories, biochemical and endocrine theories, psychological and social theories. Genetic research and the research of the action of anti-depressive medications have shown that there is significant biological background of depression. The risk of depression is 2-3 times higher in families with a history of depression then in overall population [2]. Depression is often followed with anxiety symptoms and it is possible to make three diagnoses: (a) depression, (b) anxiety disorder, (c) a mix of anxiety and depression depending on which symptoms dominate the clinical picture. In patients who have a very clear, typical clinical picture and also very significant psychomotor symptoms (slowness of motion and agitation) accompanied with a spectre of somatic symptoms there is melancholic depression or a depression with melancholic characteristics [3].

The criteria for diagnosis and the methods of classification have changed during last period. MKB-10 uses a prearranged list of at least 10 symptoms of depression. It divides a classification of depressive episode in four groups: without depression (less than 4 symptoms), mild (4 symptoms), moderate 
(5-6 symptoms) and strong (7 and more symptoms with or without psychotic symptoms). The symptoms must be present for at least two weeks. Patients with mild depressive episodes which are seen in the primary practice often recuperate spontaneously but these episodes can be persistent or can lead to development of a more serious form of this disease [4].

The basic obstacle in treating depression is that patients do not ask for help from the institutions responsible for protection of mental health, as well as that many cases of depression are not recognised in the primary health care institutions. From 1000 of people, out of 130 cases of depression only 80 of them will ask a doctor of general practice for help. Out of 80 who asked for doctor's help 49 are not recognised, mostly because the reason for the consultation was some somatic difficulty and not the fact that the patient has psychological problems despite of the clear presence of many symptoms [5]. Out of those who are detected as having depression one third is treated at the level of primary health care and only 1 out of 5 are directed to a check up by a specialised doctor [6]. It is clear that this will result in an unwanted state in which a huge number of people having symptoms of depression with all its personal and social consequences will not be recognised.

Anti-depressives are psychotropic medication which cure and improve depressive state. They can achieve therapeutical goal only if the combination of psychotherapy and sociotherapy are applied in the treatment. Anti-depressive medications are effective in the therapy of depressive psychosis. This effect is made in improvement of mood, the removal of fear and in decrease or removal of depressive mind [7]. Patients with depression are often not in a state to correctly apply the prescribed pharmacological therapy which leads to cessation/disruption of therapy, inadequate treatment of depression, frequent hospitalisations, incapability to work and frequent suicides. A patient who suffers from depression is often marginalised, stigmatised and inadequately taken care of. That is why a pharmacist through an interdisciplinary approach and appliance of pharmaceutical care can affect the proper selection of therapy and can also follow up of the patient, counsel him/her about the side effects and interactions and finally improve the quality of patient's life suffering from depression $[8,9]$.

The goal of this work is to examine the effect of pharmaceutical care on important parameters in treatment of depression and on quality of life.

\section{Methods}

\subsection{Patients}

The patients are adults 18 to 75 years old who suffer from diagnosed depressive episodes (according to the criteria set up for the diagnosis) and who are treated in General Hospital Tesanj (Bosnia and Herzegovina).

The criteria of inclusion are patients with:

- mild depressive episode (at least 4 symptoms: at least 2 typical +2 other symptoms +mild symptom of social function);

- moderate depressive episode (at least 5 symptoms: 2 typical +3 other symptoms + moderate symptom of social behaviour function)

- sever depressive episode without psychotic symptoms (at least 7 symptoms: 3 typical symptoms + at least 4 others, out of which some are extremely severe);

- patients in the study should be treated with at least one pharmacological therapy of depressive episode

\subsection{Criteria of Exclusion}

Patients with severe depressive episode with psychotic symptoms (delusions, hallucinations or depressive stupor) are not included in the study. Patients who have a history of bipolar disorder, a history of drug abuse or alcohol and cognitively challenged patients will be excluded from the study. 
The number of patients in the study is 48 . Prior to inclusion patients signed their voluntary consent for taking part in this research. The basic group of patients is divided into intervention group (23 patients) and standard group (25 patients). The intervention group is also checked by a pharmacist while the standard group only make standard appointments with a psychiatrist.

\subsection{Methods}

The study is a randomised controlled clinical study in which a psychiatrist in General Hospital Tesanj randomised patients according to the methods of random selection and the criteria of inclusion and exclusion defined in the protocol. The doctor thereafter sent an intervention group to the pharmacy where they are monitored monthly in a period of six months in a program of a supportive pharmaceutical care. As part of a program of pharmaceutical care patients have filled questionnaires about their quality of life. The pharmacist educated patients about depression medications, counselled patients about anti-depressive therapy, made notes about problems caused by the therapy with a special attention on side effects and interaction with other medications. The pharmacist applied the world's guidelines for therapy

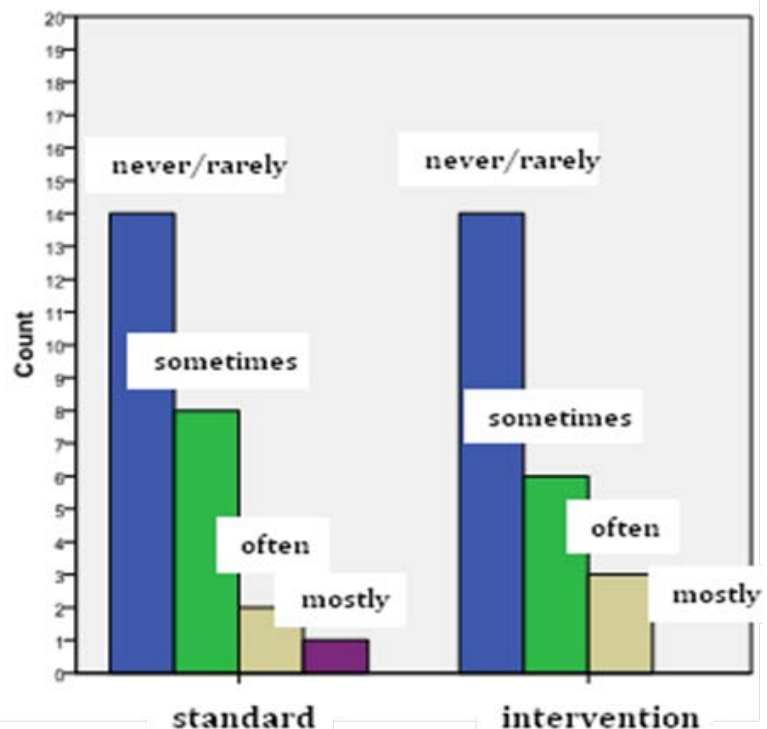

Fig. 1 The number of patients answering the question "I have nightmares" between groups after the first visit (left) and after six months (right). of depression [10]. The standard group filled only the questionnaire about quality of life during their regular visit to the doctor at the beginning of the study, after three months and after six months of the study.

\subsection{Statistical Analysis}

The data are analysed with the SPSS software for statistical analysis of data and include qualitative and quantitative elements. Ordinal categorical data are used. A comparison of the basic characteristics between the questioned groups is done using Wilcox's test of rank. Categorical variables are shown as a proportion and are analysed with hi-square test. $\mathrm{P}$ value $<0.05$ is considered as significant

\section{Results}

In the intervention group included were $69.5 \%(\mathrm{~N}=$ 16) women and $30.5 \%(\mathrm{~N}=7)$ men, while in the standard group the ratio was $84 \%(\mathrm{~N}=21)$ women and $16 \%(\mathrm{~N}=4)$ men. The average age of the patients was $55.46 \pm 14.34$, the youngest patient was 22 year old while the oldest was 80 . The gender ratio is in accordance with the World Health Organisation data.

The first question in the questionnaire considered anxiety test and results on the question "I have nightmares” are presented in Fig. 1. During the first visit

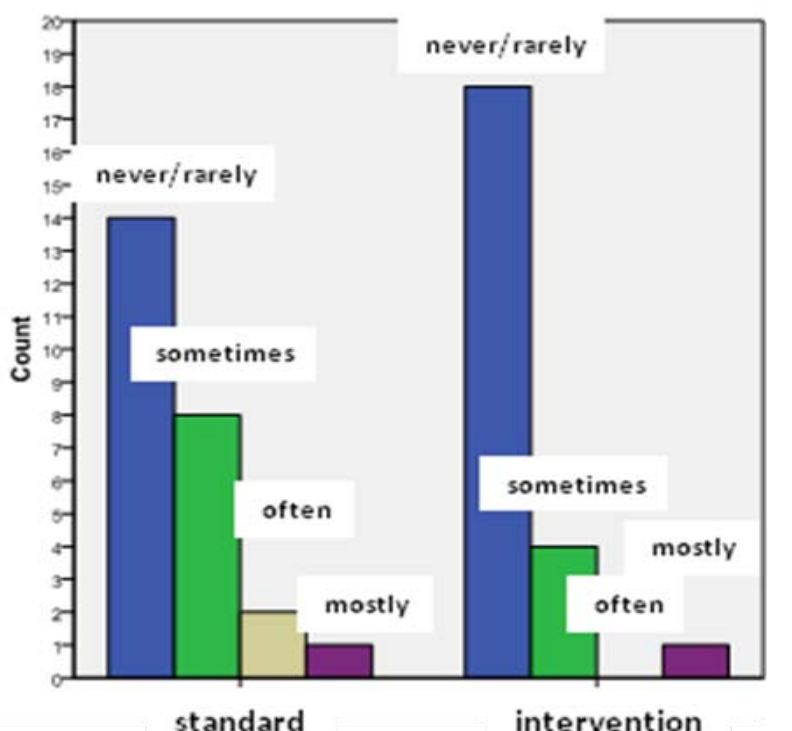


to the doctor $61 \%$ of patients in the intervention group responded that they never or seldom have nightmares. After six months, with the cooperation of the pharmacist, number of the patients who seldom have nightmare significantly increased (to 78\%). There is no variation in answers during the visits in the standard group. The statistical analysis has shown that there is a significant influence of pharmacists on the treatment of patients $(\mathrm{p}<0.05)$.

There is an evident progress in the patients who were treated with the participation of a pharmacist during six months in comparison to the standard group without the participation of a pharmacist. Also, the number of patients who sometimes or never have nightmare has decreased.

Fig. 2 shows answers on the questions if patients have "Feeling of restlessness or despair" as part of second set of questions considering quality of life. There is a slight fluctuation in the responses in the standard group during the study, while in the intervention group there is an evident difference of about 21\% in answers between the first and third visit and that smaller number of patients in the intervention group fell restlessness or despair after 6 months.

By considering the questions from the life quality

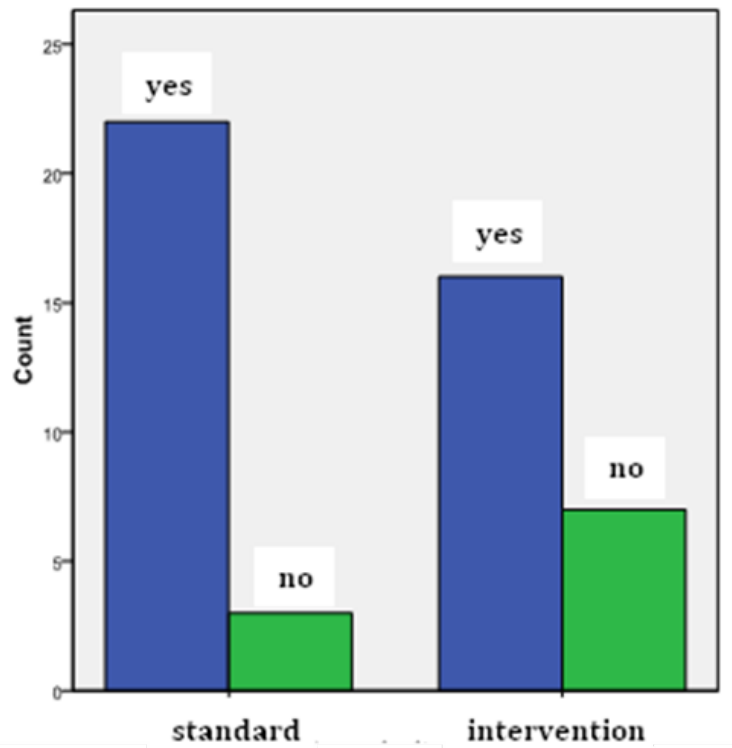

test it is visible that in comparison to the standard way of treatment (without the participation of the pharmacist), the treatment with the participation of the pharmacist showed an evident progress of the patients between the first and the third visit after 6 months.

The next question in the questionnaire considered feeling of dispiritedness, depression or hopelessness and results are presented in Fig. 3. It is shown that there is no difference between patients in the standard group at the beginning and the end of study, while in the intervention group there is a difference noticed between patients. The number of patients who rarely feel depressed increased for $29 \%$ during 6 months of the study.

The treatment with the participation of the pharmacist (intervention group) showed an evident progress of the patients after three visits. The patients feel less depressed whereas in the standard group (treatment without the participation of the pharmacist) there is no evident difference in response during 6 month of the study.

By analysing answer on the question if patients have "Poor interest or pleasure in doing something" presented on Fig. 4 it can be noticed that there is no difference in responses for the standard group, while in

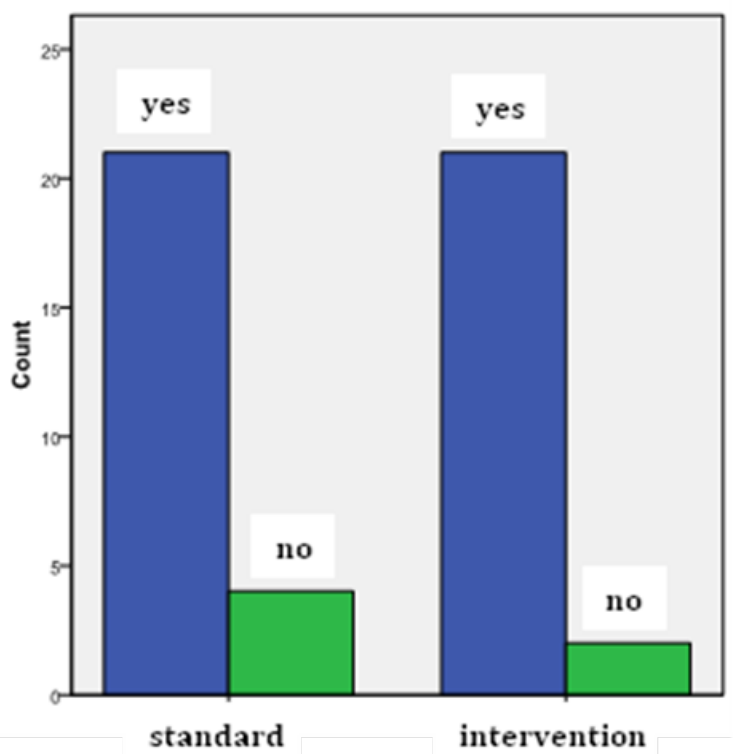

Fig. 2 The number of patients answering the question "Feeling of restlessness or despair" between groups after the first visit (left) and after six months (right). 

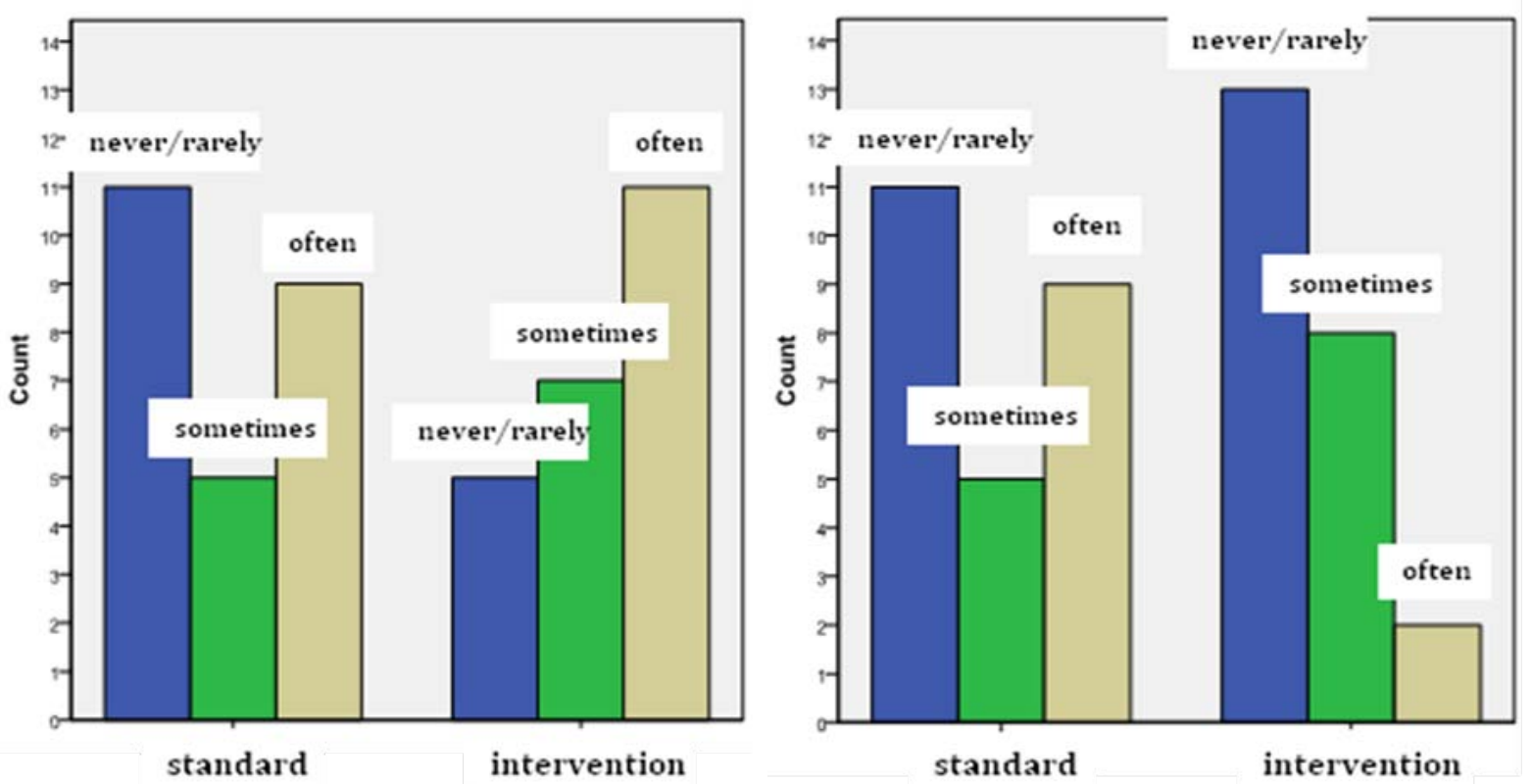

Fig. 3 The number of patients answering the question "Feeling of dispiritedness, depression or hopelessness" between groups after the first visit (left) and after six months (right).
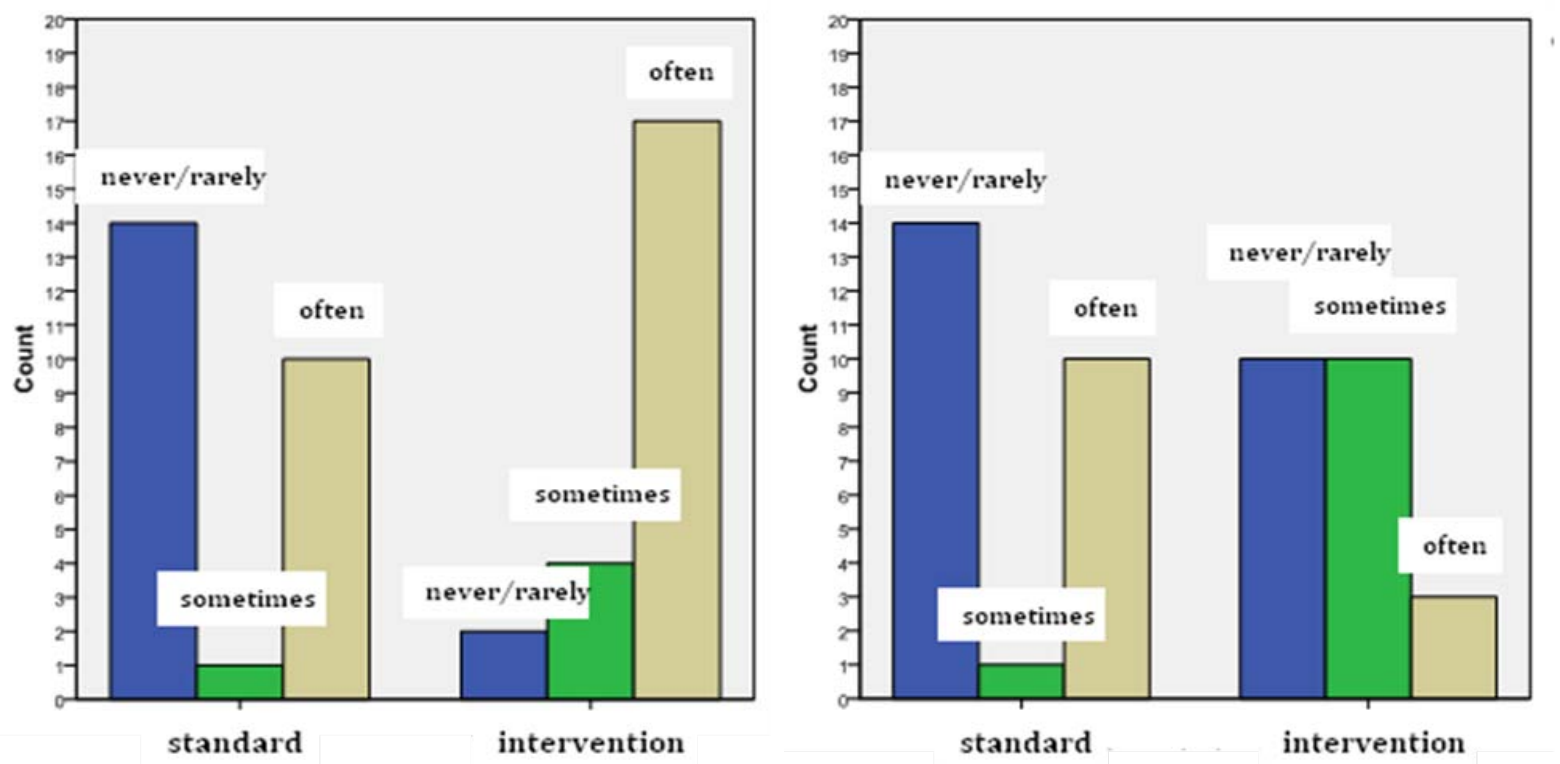

Fig. 4 The number of patients answering the question in experiencing "Poor interest or pleasure in doing something" between groups after the first visit (left) and after six months (right).

the intervention group there is a difference in responses during the study. The number of patients who never or sometimes have poor interest in doing something increased during the study in intervention group. Also, the patients have shown more interest and pleasure in doing something.

Fig. 5 shows that there is some difference for the standard group in the responses on the question if patients have "Low opinion about themselves or the feeling that they are a failure or that they have disappointed their family". In the intervention group it is evident that the largest difference is in the responses never between the first and the last visit. There is an evident progress in treatment when the pharmacist is included during the study and the patients have a better opinion of themselves. 

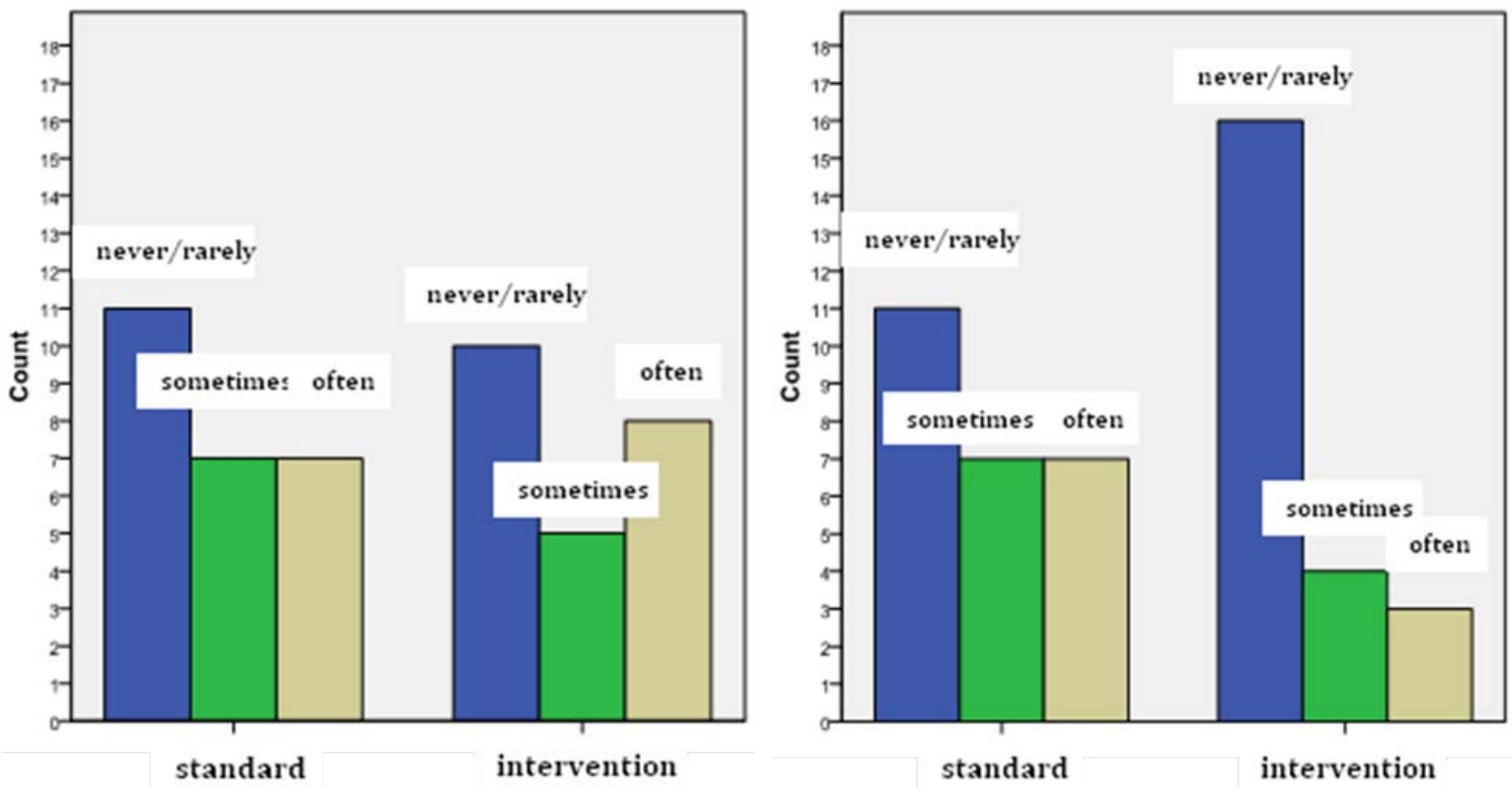

Fig. 5 The number of patients answering if they have "Low opinion about themselves or the feeling that they are a failure or that they have disappointed their family” between groups after the first visit (left) and after six months (right).

\section{Discussion}

Depression is one of the most present mental disorders and the treatment is very costly [11]. Characteristics of the patients suffering from depression are bad mood, low self-esteem and loss of interest or pleasure in activities that were once enjoyed. Also, patients from this category are frequently unable to properly apply the prescribed therapy which as a consequence can have termination of therapy, inadequate treatment of depression, frequent need for hospitalisation and even suicides. It is important to stress that depression is considered when the symptoms last continuously for two or more weeks. Persons suffering from depression are prone to underestimate and blame themselves. During the depressive episode they have troubles concentrating and their memory is also weakened. Pharmaceutical treatment of depression demands a proper selection of medications according to the global guidelines, careful patient monitoring as well as control of side effects and interactions. In Bosnia and Herzegovina there is high prevalence of depression and psychiatrists are often overloaded with patients [12]. Therefore, patients that suffer from depression are often marginalised, stigmatised and inadequately treated. Inclusion of pharmacist in the treatment of patients can influence an adequate selection of a therapy through an interdisciplinary approach and proper pharmaceutical care as well as provide monitoring of patients, counselling them about side effects and interactions, which will in the end improve the quality of life for patients that suffer from depression.

In this work a randomised controlled clinical study has been applied in which a psychiatrist from General Hospital Tešanj selected and randomised patients according to criteria for inclusion and exclusion. Contol group of patints was treated by psychiatrist only, whereas intervention group was additionally monitored by pharmacists monthly in a period of six months. All patients have filled a questionnaire. The aim of this work was to establish the influence of pharmaceutical care on the outcome of the quality of life of patients suffering from depression.

48 patients were included and monitored in this study, 11 men and 37 women. The incidence of depression is more frequent in women, which is in accordance with WHO data [13]. The aim of the study 
was to test the importance of pharmacists in an interdisciplinary team in the treatment of patients suffering from depression.

Results from applied questionnaires showed that the treatment with an interdisciplinary team which includes a pharmacist compared to a team without a pharmacist shows progress in the treatment of the depression. All tests showed the contribution of the pharmacists in the treatment of patients with depression. These results showed that the pharmacist should be included in the interdisciplinary team during the treatment of patients with depression. By further educating the pharmacist in the area of mental disorders, especially depression, even better results would be achieved. A research conducted in Belgium about the role of pharmacists in the care of patients with depression showed that pharmacists are not sufficiently involved in the treatment of patients with depression and that there is insufficient education of the pharmacists regarding mental disorders [14].

It is known that pharmacists are the most visited health professionals_-patients visit pharmacists seven times more than they visit doctors [15]. These data show that pharmacists through pharmaceutical care can make the fastest and the cheapest contribution to a good outcome of this multidisciplinary approach in the treatment of patients with depression.

\section{Conclusion}

Total 48 patients with depression treated in General Hospital Tesanj are included in the study. Intervention group with 23 patients is treated in an interdisciplinary team which included pharmacist, whereas standard group with 25 patients is treated with medical doctor only.

At the beginning of the study there is no difference in the results between the standard and the intervention group of patients. After 6 months, an evident progress in quality of life of the intervention group in the depressed patients is observed. The involvement of pharmacist in a multidisciplinary approach to the treatment of depression leads to improvement of adherence, the effect of therapy and increased quality of life.

The costs of treatment of the patients with depression are very high. These costs could be diminished if the pharmacists were included in the interdisciplinary team. It could be recommended that in the future treatment of patients with depression a pharmacist should be included.

\section{References}

[1] Kecmanović, D., Loga, S., Cerić, I., and Marković, A. 1980. Psihijatrija. Beograd-Zagreb: Medicinska knjiga.

[2] Cassano, P., and Fava, M. 2002. "Depression and Public Health: An Overview.” J. Psychosom Res. 53 (4): 849-57.

[3] Quitkin, F. M., Harrison, W., and Stewart, J. W. 1991. "Response to Phenelazine and Imipramin in Placebo Nonresponders with Atypical Depression, a New Application of the Crossover Design.” Arch. Gen. Psychiatry 48: 319-23.

[4] Brown, T. A., Campbell, L. A., and Lehman, C. L. 2001. "Current and Lifetime Comorbidity of the DSM-IV Anxiety and Mood Disorders in a Large Clinical Sample.” J. Abnormal. Psychology 110: 585-99.

[5] Kisely, S., Gater, R., and Goldberg, D. P. 1995. Results from the Manchester Centre. In Mental Illness in General Health Care: An International Study. (Eds T. B. Ustun, N. Sartorius), Chichester: Wiley, 175-91.

[6] Goldberg, D., and Huxley, P. 1980 Mental Illness in the Community: the Pathway to Psychiatric Care. London: Tavistock.

[7] Kecmanović, D. 1989. Psihijatrija tom II, Svjetlost, Sarajevo.

[8] Adler, D. A., Bungay, K. M., Wilson, I. B., Pei, Y., Supran, S., Peckham, E., Cynn, D. J., and Rogers, W. H. 2004. "The Impact of a Pharmacist Intervention on 6-Month Outcomes in Depressed Primary Care Patients." Gen Hosp. Psychiatry 26: 199-209.

[9] Finley, P. R., Rens, H. R., Pont, J. T., Gess, S. L., Louie, C., Bull, S. A., and Bero, L. A. 2002. "Impact of a Collaborative Pharmacy Practice Model on the Treatment of Depression in Primary Care.” Am. J. Health Syst. Pharm. 59: 1518-26.

[10] Drummond, M. F., Sculpher, M. J., Torrance, G. W., O'Brien. B. J., and Stoddart, G. L. 2005. Methods for the Economic Evaluation of Health Care Programmes. New York: Oxford University Press.

[11] NICE Guidelines. 2013. "Depression in adults: The treatment and management of depression in adults". 
[12] Kučukalić, A., Bravo-Mehmedbašić, A., and Džubhur-Kulenović, A. 2007. Vodič za liječenje depresivnih poremećaja. Svjetlost Sarajevo, 7-20.

[13] World Health Organization (WHO). 2002. Innovative care for chronic conditions: Building blocks for action Geneva.
[14] Sophie, L., Tim, S., Gerth, L., and Veerle F. 2012. "Pharmaceutical Care for People with Depression: Belgian Pharmacists Attitudes and Perceived Barriers." Int. J. Clin. Pharm. 34 (3): 452-9.

[15] World Health organization (WHO). 1994. Role of the Pharmacist in the Health Care System. 